\title{
THE SUBLATTICE OF AN ORTHOGONAL PAIR IN A MODULAR LATTICE
}

\author{
HERBERT GROSS and PAUL HAFNER
}

\section{Introduction}

We will be concerned with a modular lattice $\mathscr{L}$ together with an antitone mapping $\perp: \mathscr{L} \rightarrow \mathscr{L}$ such that

(1)

$$
x \leqq x^{\perp \perp} \text { for all } x \in \mathscr{L} \text {. }
$$

The following rules are easily verified:

$$
\begin{gathered}
a^{\perp \perp \perp}=a^{\perp}, \\
x \leqq y \Rightarrow x^{\perp \perp} \leqq y^{\perp \perp}, \\
(x \vee y)^{\perp}=x^{\perp} \wedge y^{\perp} .
\end{gathered}
$$

If $x=x^{\perp \perp}$ we call $x$ closed; if $x \leqq y^{\perp}$ we write $x \perp y$.

Under the assumption that $f \perp g$ we shall construct the free modular lattice $\mathscr{V}(f, g)$ generated by $\mathscr{V}(f) \cup \mathscr{V}(g)$, where $\mathscr{V}(f)$ is the orthostable lattice generated by $f \in \mathscr{L} . \mathscr{V}(f, g)$ is a distributive lattice. We will also give some conditions ensuring that $\mathscr{V}(f, g)$ or a slight modification of $\mathscr{V}(f, g)$ is orthostable. Certain special cases are studied separately because of their importance in geometry.

The value of lattice theoretical computations such as given here rests on the fact that they yield - in conjunction with certain general theorems proved in [3] and [5] - strong results on the classification of subspaces in quadratic spaces, normal bases, decomposition theorems. The role of the lattice theoretic part has been described in detail in Section 3 of [5]. Further applications of this method are given in [4]. Cf. also Remark 5 (iii) at the end. 


\section{The lattice: general case}

The $\perp$-stable lattice $\mathscr{V}(f)$ generated by an element $f \in \mathscr{L}$ (modular with $\perp$ ) is given by the following diagram

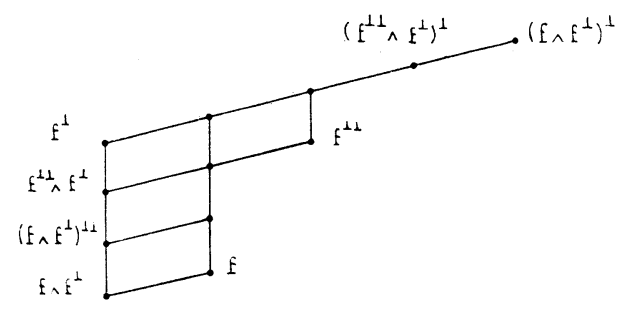

Let $\mathscr{I}(f)$ be the ideal generated in $\mathscr{V}(f)$ by $f^{\perp \perp}$; and let the filter generated by $f^{\perp}$ be denoted by $\mathscr{F}(f)$. Note that $\mathscr{V}(f)=\mathscr{I}(f) \cup \mathscr{F}(f)$ and that $\mathscr{F}(f)$ is a chain. Moreover, $\mathscr{V}(f)$ is distributive.

Considering a second element $g \in \mathscr{L}$ we prove:

Lemma 1. Assume that $f \perp g$. Then the lattice $\mathscr{V}(f, g)$ generated in $\mathscr{L}$ by $\mathscr{V}(f) \cup \mathscr{V}(g)$ is distributive.

Proof. By Theorem 6 of [6] and symmetry it suffices to verify that $\left(b \vee b^{\prime}\right) \wedge c=$ $(b \wedge c) \vee\left(b^{\prime} \wedge c\right)$ for all $b, b^{\prime} \in \mathscr{V}(f)$ and all $c \in \mathscr{V}(g)$. Since $f \perp g$ we have $y \geqq f^{\perp} \geqq g^{\perp \perp} \geqq x$ for all $x \in \mathscr{I}(g), y \in \mathscr{F}(f)$. This and the symmetric fact is expressed by

$$
\mathscr{I}(f) \leqq \mathscr{F}(g), \quad \mathscr{I}(g) \leqq \mathscr{F}(f) .
$$

The only elements in $\mathscr{V}(f)$ which are not join-irreducible are $z_{1}=f \vee\left(f \wedge f^{\perp}\right)^{\perp \perp}$, $z_{2}=f \vee\left(f^{\perp} \wedge f^{\perp \perp}\right), z_{3}=f \vee f^{\perp}, z_{4}=f^{\perp \perp} \vee f^{\perp}$. For $i=3,4$ we obtain the distributivity of $z_{i} \wedge y$ using (5) and modularity. The same works for $i=1,2$ and $y \in \mathscr{F}(g)$. Finally (5) implies that $y=f^{\perp} \wedge y$ for $y \in \mathscr{I}(g)$; therefore

$$
\begin{aligned}
& z_{1} \wedge y=\left[f \vee\left(f \wedge f^{\perp}\right)^{\perp \perp}\right] \wedge f^{\perp} \wedge y=\left(f \wedge f^{\perp}\right)^{\perp \perp} \wedge y \leqq(f \wedge y) \vee\left[\left(f \wedge f^{\perp}\right)^{\perp \perp} \wedge y\right] \\
& z_{2} \wedge y=\left[f \vee\left(f^{\perp} \wedge f^{\perp \perp}\right)\right] \wedge f^{\perp} \wedge y=f^{\perp} \wedge f^{\perp \perp} \wedge y \leqq(f \wedge y) \vee\left[\left(f^{\perp} \wedge f^{\perp \perp}\right) \wedge y\right] .
\end{aligned}
$$

This takes care of the remaining cases, bearing in mind the distributive inequality.

Remark 1. Let $\mathscr{D}$ be given by the following diagram

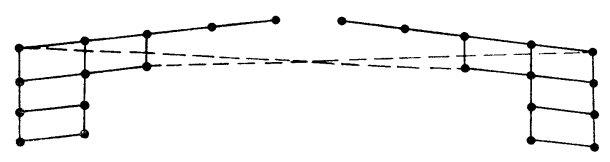

where the broken lines indicate a relation $\leqq$. The proof given above shows that the free modular lattice generated by $\mathscr{D}$ is distributive. 
A situation involving $\mathscr{D}$ appears again in the construction of the $\perp$-stable lattice generated by two elements $f, g \in \mathscr{L}, f \perp f, g \perp g$. Here

$$
\mathscr{I}_{1}=\left\{f \wedge g^{\perp}, f,\left(f \wedge g^{\perp}\right)^{\perp \perp}, f \vee\left(f \wedge g^{\perp}\right)^{\perp \perp}, f^{\perp \perp} \wedge g^{\perp}, f \vee\left(f^{\perp \perp} \wedge g^{\perp}\right), f^{\perp \perp}\right\}
$$

and

$$
\mathscr{F}_{1}=\left\{g^{\perp}, f \vee g^{\perp}, f^{\perp \perp} \vee g^{\perp},\left(f^{\perp} \wedge g^{\perp \perp}\right)^{\perp},\left(f^{\perp} \wedge g\right)^{\perp}\right\}
$$

take the place of $\mathscr{I}(f)$ and $\mathscr{F}(f)$ respectively. If $\mathscr{J}_{2}$ and $\mathscr{F}_{2}$ denote the analogous sets with $f$ and $g$ interchanged, then clearly the orthostable lattice generated by $f$ and $g$ must contain the sublattice generated by $\mathscr{J}_{1} \cup \mathscr{J}_{2} \cup \mathscr{F}_{1} \cup \mathscr{F}_{2}$ which by the proof of Lemma 1 is distributive.

In what follows we construct the free modular lattice generated by $\mathscr{D}$. We will however do it in the setup of $\mathscr{V}(f) \cup \mathscr{V}(g)$ and leave it to the reader to verify that the result has general validity.

Thanks to the distributivity of $\mathscr{V}(f, g)$ the lattice $\mathscr{V}_{2}$ generated by $\mathscr{I}(f) \cup \mathscr{I}(g)$ is the join-closure of

$$
\mathscr{I}(f) \cup \mathscr{I}(g) \cup\{x \wedge y \mid x \in \mathscr{I}(f), y \in \mathscr{I}(g)\} .
$$

As $f \perp g$ we have

$$
\{x \wedge y \mid x \in \mathscr{I}(f), y \in \mathscr{I}(g)\}=\left\{x \wedge y \mid x \in \mathscr{I}_{0}(f), y \in \mathscr{I}_{0}(g)\right\}
$$

where $\mathscr{J}_{0}(f)=\left\{f \wedge f^{\perp},\left(f \wedge f^{\perp}\right)^{\perp \perp}, f^{\perp} \wedge f^{\perp \perp}\right\}$ and similarly for $\mathscr{J}_{0}(g)$ (compare the proof of Lemma 1). Therefore, we begin by forming the free modular lattice $M$ generated by the two chains $\mathscr{J}_{0}(f), \mathscr{J}_{0}(g)$. $M$ has $8 !(4 !)^{-2}-2=68$ elements ([1] p. 66) and consists of all joins of elements out of the following diagram

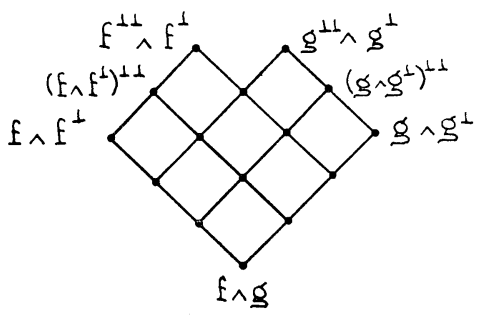

(intersections of lines represent meets of elements).

The next step is to form joins of elements in $M$ with $f, g, f \vee g, f^{\perp \perp}, g^{\perp \perp}, f \vee g^{\perp \perp}$, $f^{\perp \perp} \vee g, f^{\perp \perp} \vee g^{\perp \perp}$. This produces all elements of $\mathscr{V}_{2}$ since any element $x \vee y \vee m$, where $x \in \mathscr{I}(f), y \in \mathscr{I}(g), m \in M$ is of the form $x_{0} \vee y_{0} \vee m_{0}$ with $x_{0} \in\left\{f, f^{\perp \perp}\right\}$, $y_{0} \in\left\{g, g^{\perp \perp}\right\}, m_{0} \in M$. In an expression like $f \vee x \vee y \vee V x_{i} \wedge y_{i}$, where $x, x_{i} \in \mathscr{J}_{0}(f)$, $y, y_{i} \in \mathscr{J}_{0}(g)$ one can dispose of terms $x_{i} \wedge y_{i} \leqq f$. Thus we may assume that $x, x_{i} \neq$ $f \wedge f^{\perp}$ and hence $f \vee M=f \vee M_{1}$, where $M_{1}$ is the free modular lattice generated by the 2 chains $\left\{\left(f \wedge f^{\perp}\right)^{\perp \perp}, f^{\perp} \wedge f^{\perp \perp}\right\}$ and $\mathscr{J}_{0}(g)$. We obtain $7 !(3 ! 4 !)^{-1}-2=33$ elements or 34 elements if we include $f$. The same kind of reasoning leads to the following enumeration 
$M: 68$ elements; $f \vee M: 34$ elements (including $f$ ); $g \vee M: 34$ elements (including $g$ ); $f \vee g \vee M: 19$ elements (including $f \vee g$ ); $f^{\perp \perp} \vee M: 4$ elements (including $f^{\perp \perp}$ ); $g^{\perp \perp} \vee M: 4$ elements (including $\left.g^{\perp \perp}\right) ; f \vee g^{\perp \perp} \vee M: 3$ elements (including $f \vee g^{\perp \perp}$ ); $g \vee f^{\perp \perp} \vee M: 3$ elements (including $g \vee f^{\perp \perp}$ ); $f^{\perp \perp} \vee g^{\perp \perp}$ : 1 element.

Altogether the free modular lattice generated by $\mathscr{I}(f) \cup \mathscr{I}(g)$ has 170 elements.

The lattice $\mathscr{V}_{1}$ generated by the 2 chains $\mathscr{F}(f)$ and $\mathscr{F}(g)$ is the $\vee$-closure of the elements which are depicted in the following diagram (intersections of lines represent meets):

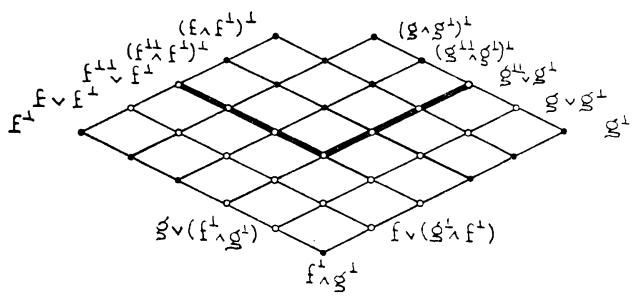

Observe that the elements marked by circles are of the form $f \vee x, f^{\perp \perp} \vee x$, $g \vee y, g^{\perp \perp} \vee y$ for $x \in\left\{f^{\perp}, f^{\perp} \wedge a \mid a \in \mathscr{F}(g)\right\}, y \in\left\{g^{\perp}, g^{\perp} \wedge b \mid b \in \mathscr{F}(f)\right\}$. Moreover, for

we have

$$
\begin{gathered}
r \in\left\{f^{\perp}, f^{\perp} \wedge\left(g \wedge g^{\perp}\right)^{\perp}, f^{\perp} \wedge\left(g^{\perp \perp} \wedge g^{\perp}\right)^{\perp}\right\}, \\
s \in\left\{g^{\perp}, g^{\perp} \wedge\left(f \wedge f^{\perp}\right)^{\perp}, g^{\perp} \wedge\left(f^{\perp \perp} \wedge f^{\perp}\right)^{\perp}\right\} \\
r \vee s=\left(r \vee f^{\perp \perp}\right) \vee\left(s \vee g^{\perp \perp}\right) .
\end{gathered}
$$

As a consequence $\mathscr{V}_{1}$ is the lattice generated by the two chains

and

$$
\left\{f^{\perp \perp} \vee f^{\perp},\left(f^{\perp \perp} \wedge f^{\perp}\right)^{\perp},\left(f \wedge f^{\perp}\right)^{\perp}\right\}
$$

$$
\left\{g^{\perp \perp} \vee g^{\perp},\left(g^{\perp \perp} \wedge g^{\perp}\right)^{\perp},\left(g \wedge g^{\perp}\right)^{\perp}\right\}
$$

together with the 20 elements below the solid line in the diagram. The total number of elements in $\mathscr{V}_{1}$ is therefore at most $68+20=88$.

Finally we prove that $\mathscr{V}_{1} \cup \mathscr{V}_{2}$ is a lattice by showing that $x \vee y$ and $x \wedge y$ are in $\mathscr{V}_{1} \cup \mathscr{V}_{2}$ whenever $x \in \mathscr{V}_{1}, y \in \mathscr{V}_{2}$. As for the joins it suffices to show that $x \vee y \in \mathscr{V}_{1}$ for $x \in \mathscr{V}_{1}$ and $y$ join-irreducible in $\mathscr{V}_{2}, y=f^{\perp} \wedge g^{\perp}$. Since $f^{\perp} \wedge g^{\perp} \wedge\left(f^{\perp \perp} \vee g^{\perp \perp}\right)=$ $\left(f^{\perp} \wedge f^{\perp \perp}\right) \vee\left(g^{\perp} \wedge g^{\perp \perp}\right)$ the only such $y$ are $f, f^{\perp \perp}, g, g^{\perp \perp}$; for these, however, the claim is obvious. Owing to distributivity we will now consider only those meets $x \wedge y$ for which $x \in \mathscr{V}_{1}, y \in \mathscr{V}_{2}$ are join-irreducible with $x \neq f^{\perp \perp} \vee g^{\perp \perp} \vee\left(f^{\perp} \wedge g^{\perp}\right)$ and $y \neq f^{\perp} \wedge g^{\perp}$. This means that

$$
\begin{aligned}
x \in\left\{f^{\perp}, f^{\perp} \wedge\left(g \wedge g^{\perp}\right)^{\perp}, f^{\perp} \wedge\left(g^{\perp} \wedge g^{\perp \perp}\right)^{\perp}, f^{\perp} \wedge g^{\perp}, g^{\perp} \wedge\left(f^{\perp} \wedge f^{\perp \perp}\right)^{\perp},\right. & \\
& \left.g^{\perp} \wedge\left(f \wedge f^{\perp}\right)^{\perp}, g^{\perp}\right\}
\end{aligned}
$$


and

These verifications are easy.

$$
y \in\left\{f, f^{\perp \perp}, g, g^{\perp \perp}\right\}
$$

We summarize:

Theorem 1. The free modular lattice generated by $\mathscr{D}$ has 258 elements.

\section{The lattice: some special cases}

We recall that $f \perp g$ is assumed throughout. From this it follows that $f^{\perp \perp} \wedge g^{\perp \perp}=$ $f^{\perp} \wedge f^{\perp \perp} \wedge g^{\perp} \wedge g^{\perp \perp}$. The following condition requires that $f^{\perp \perp} \wedge g^{\perp \perp}$ is even smaller :

$$
f^{\perp \perp} \wedge g^{\perp \perp}=\left(f \wedge f^{\perp}\right)^{\perp \perp} \wedge\left(g \wedge g^{\perp}\right)^{\perp \perp}
$$

Under this assumption

$$
\mathscr{V}_{2}=\mathscr{I}(f) \cup \mathscr{I}(g) \cup\{x \vee y \mid x \in \mathscr{I}(f), y \in \mathscr{I}(g)\} \cup \mathscr{W},
$$

where $\mathscr{W}$ is the set containing the following 17 elements:

$1 f \wedge g$

$2\left(f \wedge g^{\perp \perp}\right) \vee\left(g \wedge f^{\perp \perp}\right)$

$3 f^{\perp \perp} \wedge g^{\perp \perp}$

$4\left(f \wedge f^{\perp}\right) \vee\left(g \wedge g^{\perp}\right) \vee\left(f^{\perp \perp} \wedge g^{\perp \perp}\right)$

$5 f \vee g \vee f \perp \perp \wedge g^{\perp \perp}$

$6 f \wedge g^{\perp \perp}$

$7 g \wedge f \perp \perp$

$8\left(f \wedge f^{\perp}\right) \vee g \wedge f^{\perp \perp}$

$9\left(g \wedge g^{\perp}\right) \vee f \wedge g^{\perp \perp}$
$10 f \vee\left(g \wedge f^{\perp \perp}\right)$

$11 g \vee\left(f \wedge g^{\perp \perp}\right)$

$12\left(f \wedge f^{\perp}\right) \vee\left(f^{\perp \perp} \wedge g^{\perp \perp}\right)$

$13\left(g \wedge g^{\perp}\right) \vee\left(f^{\perp \perp} \wedge g^{\perp \perp}\right)$

$14 f \vee(f \perp \perp \wedge g \perp \perp)$

$15 g \vee\left(f \perp \perp \wedge g^{\perp \perp}\right)$

$16 f \vee\left(g \wedge g^{\perp}\right) \vee\left(f^{\perp \perp} \wedge g^{\perp \perp}\right)$

$17 g \vee\left(f \wedge f^{\perp}\right) \vee\left(f^{\perp \perp} \wedge g^{\perp \perp}\right)$.

To prove (7) note that by distributivity $\mathscr{V}_{2}$ consists of joins $u_{1} \vee u_{2} \vee u_{3} \vee \ldots \vee u_{r}$, where (a) $u_{i} \in \mathscr{I}(f) \cup \mathscr{I}(g)$ or (b) $u_{i}$ is a meet $x \wedge y$ of join-irreducible elements $x \in \mathscr{I}(f)$, $y \in \mathscr{I}(g)$. From (6) and $f \perp g$ we see that the joins of elements of type (b) form the set

$$
\mathscr{V}=\left\{f^{\perp \perp} \wedge g^{\perp \perp},\left(f^{\perp \perp} \wedge g\right) \vee\left(g^{\perp \perp} \wedge f\right), f^{\perp \perp} \wedge g, g^{\perp \perp} \wedge f, f \wedge g\right\} .
$$

Under the assumption (6) $\mathscr{V}_{2}$ therefore has at most $63+17=80$ elements.

Condition (6), which does not have any bearing on $\mathscr{V}_{1}$, can be obtained from

$$
f^{\perp} \vee g^{\perp}=\left(f \wedge f^{\perp}\right)^{\perp} \vee\left(g \wedge g^{\perp}\right)^{\perp}
$$

by applying $\perp$. Equation (8) has very strong consequences: 
Lemma 2. Assume that $f \perp g$ and that (8) holds. Then

$$
\left(y_{1} \wedge y_{2}\right) \vee\left(y_{1}^{\prime} \wedge y_{2}^{\prime}\right)=\left(y_{1} \vee y_{1}^{\prime}\right) \wedge\left(y_{2} \vee y_{2}^{\prime}\right)
$$

for all $y_{1}, y_{1}^{\prime} \in \mathscr{F}(f)$ and all $y_{2}, y_{2}^{\prime} \in \mathscr{F}(g)$. In particular $\mathscr{F}(f) \cup \mathscr{F}(g) \cup$ $\{x \wedge y \mid x \in \mathscr{F}(f), y \in \mathscr{F}(g)\} \cup\left\{f^{\perp} \vee g^{\perp}\right\}$ is $\vee$-closed and hence a sublattice of $\mathscr{L}$, i.e. it is $\mathscr{V}_{2}$; card $\mathscr{V}_{2} \leqq 36$.

Proof. It is clear that $\leqq$ holds in (9). To obtain the converse inclusion we consider the case $y_{1} \leqq y_{1}^{\prime}$ and $y_{2}^{\prime} \leqq y_{2}$ (the other cases being trivial). The right hand side of (9) then becomes

$$
\begin{aligned}
y_{1}^{\prime} \wedge y_{2} & =y_{1}^{\prime} \wedge y_{2} \wedge\left[\left(f \wedge f^{\perp}\right)^{\perp} \vee\left(g \wedge g^{\perp}\right)^{\perp}\right]=y_{1}^{\prime} \wedge y_{2} \wedge\left(f^{\perp} \vee g^{\perp}\right) \\
& =\left(f^{\perp} \wedge y_{2}\right) \vee\left(g^{\perp} \wedge y_{1}^{\prime}\right) \leqq\left(y_{1} \wedge y_{2}\right) \vee\left(y_{1}^{\prime} \wedge y_{2}^{\prime}\right) .
\end{aligned}
$$

This proves the lemma.

Theorem 2. Let $\mathscr{L}$ be a modular lattice with a Galois autoconnection $\perp$. If $f \perp g$ and (6) holds then card $\mathscr{V}(f, g) \leqq 168$ and $\mathscr{V}(f, g)=\mathscr{V}_{1} \cup \mathscr{V}_{2}$, where $\mathscr{V}_{2}$ is given by (7) and $\mathscr{V}_{1}$ is generated by two chains. If instead of (6) one assumes (8), then $\mathscr{V}_{2}$ is as before, $\mathscr{V}_{1}$ is the product of 2 chains and card $\mathscr{V}(f, g) \leqq 116$.

Remark 2. The same considerations are valid in the case $f \perp f, g \perp g$ provided (6) is replaced by and (8) is replaced by

It is easily seen that

$$
f^{\perp \perp} \wedge g^{\perp \perp}=\left(f \wedge g^{\perp}\right)^{\perp \perp} \wedge\left(g \wedge f^{\perp}\right)^{\perp \perp}
$$

implies (8) if $f \perp g$ and also implies (11) if $f \perp f, g \perp g$.

\section{Orthostability}

We want to be sure that $x^{\perp} \in \mathscr{V}(f, g)$ for all $x \in \mathscr{V}(f, g)$. Since $(a \vee b)^{\perp}=$ $a^{\perp} \wedge b^{\perp}$ and $\mathscr{V}(f, g)$ is a lattice we need only find the orthogonals of join-irreducible elements. If $x \in \mathscr{\mathscr { V }}$ is join-irreducible, then $x \in \mathscr{F}(f)$, or $x \in \mathscr{F}(g)$, or $x=u^{\perp} \wedge v^{\perp}$ for some $u \in \mathscr{I}(f), v \in \mathscr{I}(g)$. In the latter case $x^{\perp}=(u \vee v)^{\perp \perp}$ and the following condition must be satisfied for $x^{\perp}$ to belong to $\mathscr{V}(f, g)$

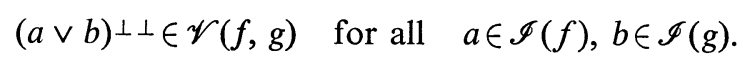

Another problem appears when we check orthogonals of elements in $\mathscr{V}_{2}: f \wedge g=\left(f \wedge f^{\perp}\right) \wedge\left(g \wedge g^{\perp}\right) \leqq\left(f \wedge f^{\perp}\right)^{\perp \perp} \wedge\left(g \wedge g^{\perp}\right)^{\perp \perp}$ and therefore $(f \wedge g)^{\perp} \geqq$ $\left[\left(f \wedge f^{\perp}\right)^{\perp} \vee\left(g \wedge g^{\perp}\right)^{\perp}\right]^{\perp \perp}$. Since $\left(f \wedge f^{\perp}\right)^{\perp} \vee\left(g \wedge g^{\perp}\right)^{\perp}$ is the largest element of 
$\mathscr{V}(f, g)$, this lattice will have to be extended at the top end unless $\left(f \wedge f^{\perp}\right)^{\perp} \vee$ $\left(g \wedge g^{\perp}\right)^{\perp}$ is closed and $(f \wedge g)^{\perp \perp}=\left(f \wedge f^{\perp}\right)^{\perp \perp} \wedge\left(g \wedge g^{\perp}\right)^{\perp \perp}$. Postponing the problem of such an extension at the moment we consider only $x \in \mathscr{V}_{2}$ such that $x>\left(f \wedge f^{\perp}\right)^{\perp \perp} \wedge\left(g \wedge g^{\perp}\right)^{\perp \perp}$, or $x \geqq f \wedge f^{\perp}$, or $x \geqq g \wedge g^{\perp}$. The join-irreducible ones among them are elements of $\mathscr{I}(f)$ or $\mathscr{I}(g)$ or meets $x=a^{\perp \perp} \wedge b^{\perp \perp}$, where $a \in \mathscr{I}(f)$, $b \in \mathscr{I}(g)$. In the last case $x^{\perp}=\left(a^{\perp} \vee b^{\perp}\right)^{\perp \perp}$. Thus, a further condition must be satisfied:

(14) For $a \in \mathscr{I}(f), b \in \mathscr{I}(g)$ such that $a^{\perp} \vee b^{\perp}<\left(f \wedge f^{\perp}\right)^{\perp} \vee\left(g \wedge g^{\perp}\right)^{\perp}$ the closure of $a^{\perp} \vee b^{\perp}$ also belongs to $\mathscr{V}(f, g)$.

The only join-irreducible elements of $\mathscr{V}_{2}$ not yet considered are

$$
f \wedge g^{\perp \perp}, f \wedge\left(g \wedge g^{\perp}\right)^{\perp \perp}, f \wedge g, g \wedge\left(f \wedge f^{\perp}\right)^{\perp \perp}, g \wedge f^{\perp \perp} .
$$

To be able to deal with $f \wedge g^{\perp \perp}$ and $g \wedge f^{\perp \perp}$ we must require that

(15) $\left(f \wedge g^{\perp \perp}\right)^{\perp \perp}$ and $\left(g \wedge f^{\perp \perp}\right)^{\perp \perp}$ belong to $\mathscr{V}(f, g)$ and are comparable to $\left(f \wedge f^{\perp}\right)^{\perp \perp} \wedge\left(g \wedge g^{\perp}\right)^{\perp \perp}$.

The elements below $\left(f \wedge f^{\perp}\right)^{\perp \perp} \wedge\left(g \wedge g^{\perp}\right)^{\perp \perp}$ make it necessary to extend $\mathscr{V}(f, g)$ at the top end; again we must require that

(16) the closures of elements $\leqq\left(f \wedge f^{\perp}\right)^{\perp \perp} \wedge\left(g \wedge g^{\perp}\right)^{\perp \perp}$ belong to $\mathscr{V}(f, g)$.

Assuming (16) one can add up to 6 elements at the top end of $\mathscr{V}(f, g)$; the maximum number of 6 is needed if $\left(f \wedge f^{\perp}\right)^{\perp} \vee\left(g \wedge g^{\perp}\right)^{\perp}$ is not closed, and all four elements below $\left(f \wedge f^{\perp}\right)^{\perp \perp} \wedge\left(g \wedge g^{\perp}\right)^{\perp \perp}$ are closed:

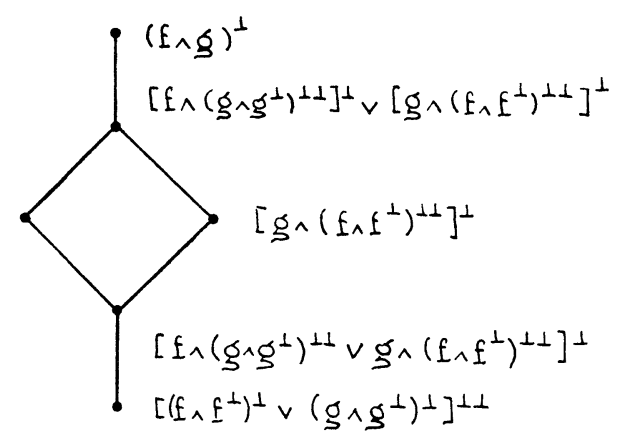

We summarize the result as a theorem:

Theorem 3. $\mathscr{V}(f, g)$ or a small extension of $\mathscr{V}(f, g)$ is orthostable provided the conditions (13), (14), (15), and (16) hold. The maximum number of elements in the orthostable lattice is 264 .

The conditions in Theorem 3 are satisfied in the following situation:

(16) closures of elements $\leqq\left(f \wedge f^{\perp}\right)^{\perp \perp} \wedge\left(g \wedge g^{\perp}\right)^{\perp \perp}$ belong to $\mathscr{V}(f, g)$.

(17) $\quad\left(f \wedge g^{\perp \perp}\right)^{\perp \perp}=\left(f \wedge f^{\perp}\right)^{\perp \perp} \wedge g^{\perp \perp} ; \quad\left(g \wedge f^{\perp \perp}\right)^{\perp \perp}=f^{\perp \perp} \wedge\left(g \wedge g^{\perp}\right)^{\perp \perp}$;

(18) $\left[\left(f \wedge f^{\perp}\right)^{\perp \perp} \vee\left(g \wedge g^{\perp}\right)^{\perp \perp}\right]^{\perp \perp}=\left(f \wedge f^{\perp}\right)^{\perp \perp} \vee\left(g \wedge g^{\perp}\right)^{\perp \perp} \vee\left(f^{\perp \perp} \wedge g^{\perp \perp}\right)$; 
(19) the following are closed:

$$
f^{\perp \perp} \vee g^{\perp \perp}, f^{\perp \perp} \vee\left(g^{\perp} \wedge g^{\perp \perp}\right), f^{\perp \perp} \vee\left(g \wedge g^{\perp}\right)^{\perp \perp}, g^{\perp \perp} \vee\left(f^{\perp} \wedge f^{\perp \perp}\right),
$$

(20) the following are closed:

$$
g^{\perp \perp} \vee\left(f \wedge f^{\perp}\right)^{\perp \perp}
$$

$$
\left(f^{\perp} \wedge f^{\perp \perp}\right)^{\perp} \vee\left(g \wedge g^{\perp}\right)^{\perp},\left(f \wedge f^{\perp}\right)^{\perp} \vee\left(g^{\perp} \wedge g^{\perp \perp}\right)^{\perp},\left(f^{\perp} \wedge f^{\perp \perp}\right) \perp \vee\left(g^{\perp} \wedge g^{\perp \perp}\right) \perp
$$

Remark 3. Note that the right hand side in (18) is closed provided (19) holds, since it is the meet of $f^{\perp \perp} \vee\left(g \wedge g^{\perp}\right)^{\perp \perp}$ and $g^{\perp \perp} \vee\left(f \wedge f^{\perp}\right)^{\perp \perp}$. Given (19), the joins $a \vee b$ of closed elements $a \in \mathscr{I}(f), b \in \mathscr{I}(g)$ are closed, being meets of elements listed in (19).

To conclude this section we return to the special cases treated in Section 2 and consider the question of orthostability. As before, $\mathscr{V}_{1}^{\perp}$ is taken care of by assuming

$$
\mathscr{V}(f, g) \text { contains }(a \vee b)^{\perp \perp} \text { for all } a \in \mathscr{I}(f), b \in \mathscr{I}(g) .
$$

The set of join-irreducible elements of $\mathscr{V}_{2}$ which are not contained in $\mathscr{I}(f) \cup \mathscr{I}(g)$ is the set $\mathscr{V}$ as defined at the beginning of Section 2. If $\left[\mathscr{V}^{\perp}\right]$ is the lattice generated by $\mathscr{V}^{\perp}$, then $\mathscr{V}_{0}=\mathscr{V}(f, g) \cup\left[\mathscr{V}^{\perp}\right]$ is a lattice because $\mathscr{V}(f, g) \leqq\left(f^{\perp \perp} \wedge g^{\perp \perp}\right)^{\perp}=$ $\left(f^{\perp} \vee g^{\perp}\right)^{\perp \perp}$ by (6). If $\mathscr{V}_{0}$ is to be $\perp$-stable we must have the elements of $\left[\mathscr{V}^{\perp}\right]^{\perp}$ in $\mathscr{V}(f, g)$; this will happen precisely when $\left(\mathscr{V}^{\perp}\right)^{\perp} \subset \mathscr{V}$. This proves

Lem ma 3. Assume (6). Then, with the notation introduced above, $\mathscr{V}(f, g) \cup\left[\mathscr{V}^{\perp}\right]$ is a lattice. This lattice is orthostable if and only if $(a \vee b)^{\perp \perp} \in \mathscr{V}(f, g)$ for all $a \in \mathscr{I}(f)$, $b \in \mathscr{I}(g)$ and

$$
(\mathscr{V} \perp)^{\perp} \subset \mathscr{V}
$$

We now prove

Lemma 4. For all $x \in \mathscr{L}$ with $f^{\perp} \leqq x^{\perp} \leqq f^{\perp} \vee g^{\perp}$ we have

$$
\left[x^{\perp \perp} \vee\left(g^{\perp \perp} \wedge f^{\perp \perp}\right)\right]^{\perp \perp}=\left(x^{\perp \perp} \vee g^{\perp \perp}\right) \perp \perp \wedge f^{\perp \perp} .
$$

Proof. We have $x^{\perp} \wedge\left(f^{\perp} \vee g^{\perp}\right)=x^{\perp} \wedge\left(f^{\perp} \vee g^{\perp}\right)^{\perp \perp}$ since both sides reduce to $x^{\perp}$ by the assumption of the lemma. By modularity the left hand side is equal to $f^{\perp} \vee\left(x^{\perp} \wedge g^{\perp}\right)=f^{\perp} \vee\left(x^{\perp} \wedge g^{\perp}\right)^{\perp \perp}$; the right hand side equals $\left[x^{\perp \perp} \vee\left(f^{\perp \perp} \wedge g^{\perp \perp}\right)\right]^{\perp}$. Taking orthogonals on both sides yields the asserted equality.

Remark 4. Obviously, if $f^{\perp} \vee g^{\perp}$ is assumed closed, then by the above proof (22) holds for all $x \in \mathscr{L}$ with $f^{\perp} \leqq x^{\perp}$.

The following lemma elaborates on the first condition ennunciated in Lemma 3:

Lemma 5. Assume that $f \perp g$ satisfy (8) and the closedness condition

$$
f^{\perp \perp \vee} g^{\perp \perp}=(f \vee g)^{\perp \perp} \text {. }
$$

Then we have

$$
x_{1}^{\perp \perp} \vee x_{2}^{\perp \perp}=\left(x_{1} \vee x_{2}\right)^{\perp \perp}
$$

for all $x_{1} \in \mathscr{I}(f), x_{2} \in \mathscr{I}(g)$. 
Proof. By (23)

$\left(x_{1}^{\perp \perp} \vee x_{2}^{\perp \perp}\right)^{\perp \perp}=\left(x_{1}^{\perp \perp} \vee x_{2}^{\perp \perp}\right)^{\perp \perp} \wedge\left(f^{\perp \perp} \vee g^{\perp \perp}\right) \leqq\left(x_{1}^{\perp \perp} \vee g^{\perp \perp}\right)^{\perp \perp} \wedge\left(f^{\perp \perp} \vee g^{\perp \perp}\right)$.

By distributivity and Lemma 4 therefore $\left(x_{1}^{\perp \perp} \vee x_{2}^{\perp \perp}\right)^{\perp \perp} \leqq\left(x_{1}^{\perp \perp} \vee\left(g^{\perp \perp} \wedge f^{\perp \perp}\right)\right)^{\perp \perp} \vee$ $g^{\perp \perp}=x_{1}^{\perp \perp} \vee g^{\perp \perp} \quad$ (the last equality by (6)). By a symmetric argumentation $\left(x_{1}^{\perp \perp} \vee x_{2}^{\perp \perp}\right)^{\perp \perp} \leqq\left(f^{\perp \perp} \vee x_{2}^{\perp \perp}\right) \quad$ so that $\left(x_{1}^{\perp \perp} \vee x_{2}^{\perp \perp}\right)^{\perp \perp} \leqq\left(x_{1}^{\perp \perp} \vee g^{\perp \perp}\right) \wedge\left(f^{\perp \perp} \vee\right.$ $\left.x_{2}^{\perp \perp}\right)=x_{1}^{\perp \perp} \vee x_{2}^{\perp \perp}$ by again using (6). Obviously, if in this proof $f^{\perp} \vee g^{\perp}$ is assumed closed then by Remark 4 we need not assume (8) in order to quote Lemma 4 . In other words, we have also proved the

Lemma 5'. Assume that $f \perp g$ has $f^{\perp \perp} \vee g^{\perp \perp}$ and $f^{\perp} \vee g^{\perp}$ closed. Then (24) holds for all $x_{1}, x_{2} \in \mathscr{L}$ with $f^{\perp \perp} \wedge g^{\perp \perp} \leqq x_{1}^{\perp \perp} \leqq f^{\perp \perp}, f^{\perp \perp} \wedge g^{\perp \perp} \leqq x_{2}^{\perp \perp} \leqq g^{\perp \perp}$.

Another possibility to obtain (24) is to require (23) and condition

$$
f^{\perp \perp} \vee\left(g \wedge g^{\perp}\right)^{\perp \perp}, g^{\perp \perp} \vee\left(f \wedge f^{\perp}\right)^{\perp \perp} \quad \text { are closed; }
$$

for, simple calculations show that (23) and (25) imply closedness of all spaces $x_{1}^{\perp \perp} \vee x_{2}^{\perp \perp}$ occuring in (24).

In order to satisfy (21) we may require condition

$$
f \perp \perp \wedge g^{\perp \perp}=(f \wedge g) \perp \perp
$$

- which means that the lattice $\mathscr{V}^{\perp}$ of Lemma 3 reduces to $\left\{\left(f^{\perp} \vee g^{\perp}\right)^{\perp \perp}\right\}$ - or

$$
f \wedge g, f \wedge g^{\perp \perp}, g \wedge f^{\perp \perp},\left(f \wedge g^{\perp \perp}\right) \vee\left(g \wedge f^{\perp \perp}\right) \text { are closed, }
$$

which means that the elements of $\mathscr{V}$ are closed so that $\perp: \mathscr{V} \rightarrow \mathscr{V}^{\perp}$ is a bijection. Notice that (26) implies (6). We summarize:

Theorem 4. Let $\mathscr{L}$ be a modular lattice equipped with a Galois autoconnection $\perp$. Assume that $f, g \in \mathscr{L}$ satisfy $f \perp g$. Let $\mathscr{V}(f, g)$ be the sublattice generated by the set $\mathscr{V}(f) \cup \mathscr{V}(g)$, where $\mathscr{V}(f), \mathscr{V}(g)$ are the $\perp$-stable sublattices generated by $f$ and $g$ respectively. In order that the $\perp$-stable lattice $\mathscr{V}(f, g, \perp)$ generated by $\mathscr{V}(f) \cup \mathscr{V}(g)$ (i.e. the $\perp$-stable lattice generated by $\{f, g\})$ is finite and distributive either of the following four conditions is sufficient: (26) \& (23) \& (25), (8) \& (23) \& (26), (6) \& (23) \& (25) \& (27), (8) \& (23) \& (27). We then have $\mathscr{V}(f, g, 1)=\mathscr{V}(f, g) \cup$ $\left\{\left(f^{\perp} \vee g^{\perp}\right)^{\perp \perp}\right\}$ in the first two cases and

$$
\begin{aligned}
\mathscr{V}(f, g, \perp)=\mathscr{V}(f, g) \cup & \left\{\left(f^{\perp} \vee g^{\perp}\right)^{\perp \perp},\left(f \wedge g^{\perp \perp}\right)^{\perp} \wedge\left(g \wedge f^{\perp \perp}\right)^{\perp},\left(f \wedge g^{\perp \perp}\right)^{\perp},\right. \\
& \left.\left(g \wedge f^{\perp \perp}\right)^{\perp},\left[f \wedge\left(g \wedge g^{\perp}\right)^{\perp \perp}\right]^{\perp} \vee\left[g \wedge\left(f \wedge f^{\perp}\right)^{\perp \perp}\right]^{\perp},(f \wedge g)^{\perp}\right\}
\end{aligned}
$$

in the last two cases. Upper bounds for the cardinality of $\mathscr{V}(f, g, \perp)$ in the four cases listed are respectively 169, 117, 174, 122; they are attained in the "free" cases.

Theorem 5. Assume that $f \perp g$ has $f^{\perp \perp} \vee g^{\perp \perp}$ and $f^{\perp} \vee g^{\perp}$ closed. Then $\left(f^{\perp \perp}, g^{\perp \perp}\right)$ is a modular and dual modular pair in the lattice $\mathscr{L}_{\perp \perp}$ of all closed elements of $\mathscr{L}$. If in addition (8) and (26) resp. (8) and (27) are assumed, then $\mathscr{V}(f, g, \perp)$ is distributive and has at most 116 resp. 121 elements. 
Proof. By Remark $3\left(f^{\perp \perp}, g^{\perp \perp}\right)$ is a modular pair in $\mathscr{L}_{\perp \perp}$; in order to show that it is a dual modular pair we have to prove that $\left(\left(z \wedge f^{\perp \perp}\right) \vee g^{\perp \perp}\right)^{\perp \perp}=$ $z \wedge\left(f^{\perp \perp} \vee g^{\perp \perp}\right)^{\perp \perp}$ for all $z \geqq g^{\perp \perp}$ in $\mathscr{L}_{\perp \perp}$. Since $f^{\perp \perp} \vee g^{\perp \perp}$ is closed and $\mathscr{L}$ is modular the right hand side is $\left(z \wedge f^{\perp \perp}\right) \vee g^{\perp \perp}$. In order to show that this is closed we quote Lemma $5^{\prime}$ with $x_{2}=g^{\perp \perp}, x_{1}=z \wedge f^{\perp \perp}$. Cardinalities for $\mathscr{V}(f, g, \perp)$ follow from Theorem 4 .

Remark 5. (i) See Theorem (33.4) in [7] for modular and dual modular pairs in hermitean spaces. (ii) We have constructed sesquilinear spaces $E$ with subspaces $F, G$ such that (23) \& (26) \& (8) resp. (23) \& (27) \& (8) is satisfied and such that all 117 resp. 122 elements of $\mathscr{V}(F, G, \perp)$ are different. (iii) Let $E$ be a vector space equipped with a non degenerate alternate form, $\operatorname{dim} E=\aleph_{0}$ and $F, G$ subspaces with $F \cap G=(0), F^{\perp \perp}+G^{\perp \perp}$ closed and $F^{\perp}+G^{\perp}=E$. Brand [2] gave a recursive construction for an orthogonal decomposition of $E, E=E_{1} \oplus E_{2}$, such that $F \subset E_{1}$, $G \subset E_{2}$. From this geometric result it follows readily that the lattice $\mathscr{V}(F, G, \perp)$ is given by $\mathscr{I}(F) \cup \mathscr{I}(G) \cup(\mathscr{I}(F) \vee \mathscr{I}(G)) \cup \mathscr{F}(F) \cup \mathscr{F}(G) \cup(\mathscr{F}(F) \wedge \mathscr{F}(G))$, in particular $\mathscr{V}(F, G, \perp)$ is distributive and has 98 elements. The fruitfulness of the method hinted at in Introduction is based on a reversal of steps: First $\mathscr{V}(F, G, \perp)$ is computed, then the theorems of [3] are applied in order to conclude that $E$ must split in the manner indicated.

\section{References}

[1] Birkhoff, G.: Lattice theory. - American Mathematical Society Colloquium Publications, XXV, Providence, R. I., 1973.

[2] BRAND, L.: Erweiterung von algebraischen Isometrien in sesquilinearen Räumen. - Universität Zürich, Dissertation, 1974.

[3] Gross, H.: Isomorphisms between lattices of linear subspaces which are induced by isometries. J. Algebra 49, 1977, 537-546.

[4] Gross, H.: Untersuchungen über quadratische Formen in Körpern der Charakteristik 2. J. Reine Angew. Math. 297, 1978, 80-91.

[5] Gross, H., and H. A. Keller: On the non trace-valued forms. - To appear in Advances in Math.

[6] JoŃsson, B.: Distributive sublattices of a modular lattice. - Proc. Amer. Math. Soc. 6, 1955, 682-688.

[7] Maeda, E., and S. Maeda: Theory of symmetric lattices. - Die Grundlehren der mathematischen Wissenschaften 173. Springer-Verlag, Berlin-Heidelberg-New York, 1970.

Universität Zürich

Mathematisches Institut

CH-8032 Zürich

Switzerland
University of Auckland

Department of Mathematics

Auckland

New Zealand

Received 22 December 1977 